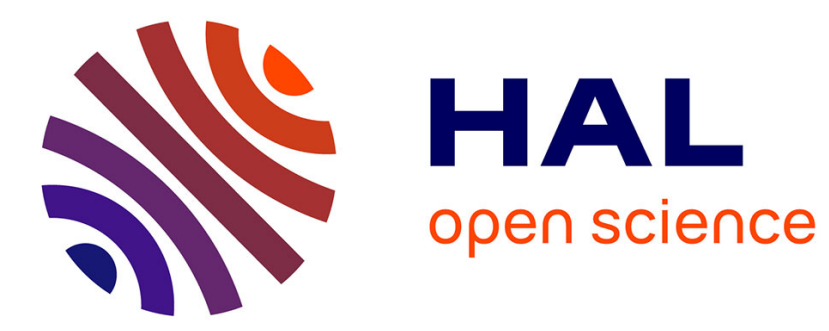

\title{
Evidence for Diverging Barriers in the Disordered Vortex Solid in the K,Ba BiO3 Superconducting Oxide
}

\author{
I. Joumard, Thierry Klein, J. Marcus
}

\section{To cite this version:}

I. Joumard, Thierry Klein, J. Marcus. Evidence for Diverging Barriers in the Disordered Vortex Solid in the K,Ba BiO3 Superconducting Oxide. Physical Review Letters, 2001, 87, pp.167002. 10.1103/PhysRevLett.87.167002 . hal-00959106

\section{HAL Id: hal-00959106 https://hal.science/hal-00959106}

Submitted on 13 Mar 2014

HAL is a multi-disciplinary open access archive for the deposit and dissemination of scientific research documents, whether they are published or not. The documents may come from teaching and research institutions in France or abroad, or from public or private research centers.
L'archive ouverte pluridisciplinaire HAL, est destinée au dépôt et à la diffusion de documents scientifiques de niveau recherche, publiés ou non, émanant des établissements d'enseignement et de recherche français ou étrangers, des laboratoires publics ou privés. 


\title{
Evidence for Diverging Barriers in the Disordered Vortex Solid in the $(\mathrm{K}, \mathrm{Ba}) \mathrm{BiO}_{3}$ Superconducting Oxide
}

\author{
I. Joumard, T. Klein, and J. Marcus \\ Laboratoire d'Etudes des Propriétés Electroniques des Solides, Centre National de la Recherche Scientifique, BP 166, \\ 38042 Grenoble Cedex 9, France \\ (Received 20 March 2001; published 26 September 2001)
}

\begin{abstract}
Vortex dynamics has been investigated in the cubic $(\mathrm{K}, \mathrm{Ba}) \mathrm{BiO}_{3}$ superconductor using ac susceptibility measurements on a large frequency range $(0.03 \mathrm{~Hz}<\omega<60 \mathrm{kHz})$. Power law diverging barriers have been obtained on both sides of the order-disorder transition line. The $\mu$ exponent remains close to $5 / 2$ (elastic creep value) in some part of the disordered phase and finally decreases at high temperature and/or high field, in good agreement with the recent plastic collective creep theory [J. Kierfeld, H. Nordborg, and V. M. Vinokur, Phys. Rev. Lett., 85, 4948 (2000)].
\end{abstract}

DOI: 10.1103/PhysRevLett.87.167002

PACS numbers: 74.60.Ge, 74.25.Nf

One of the most complex phenomena observed in high $T_{c}$ oxides is the nonlinear response of vortices to a driving force. For small driving currents (i.e., smaller than the critical current), vortices can hardly overcome current dependent creeping barriers by thermal activation leading to highly non linear current-voltage characteristics. In the presence of randomly distributed point defects (so-called collective creep model [1]) the elastic structure of the vortex lattice is expected to lead to diverging barriers of the form $U_{e l}(j) \propto j^{-\mu}$, where $\mu$ depends on the size of the creeping vortex bundle [2,3]. Strong evidence for those diverging barriers in the ordered phase has been obtained both by magnetic relaxation $[4,5]$ and by transport measurements [6] (for a review, see also [7]).

However, it has been suggested that the ordered vortex solid transforms into a highly disordered vortex glass above some transition field $B^{*}(T)[3,8]$ and the description of the creep phenomenom in this phase remains an open question. In particular, it is not clear whether the collective creep formalism can still be used in this phase or not. Indeed, it has been suggested by Abulafia et al. [9] that plastic vortex creep should be associated with a noncollective motion of dislocations in the vortex lattice (by analogy with dislocated solids). The authors thus proposed that the so-called fishtail effect in $\mathrm{YBaCuO}$ crystals could correspond to a crossover in the flux dynamics from an elastic to a plastic creep regime in which the activation energy would be described by a nondiverging barrier: $U_{p l}^{n c}(j) \propto 1-\sqrt{j / j_{p l}^{0}}$ (where $j_{p l}^{0}$ is the critical current for plastic motion). A similar formalism was then used in order to describe the creep mecanism in the vicinity of the peak effect in various cuprates [10].

On the other hand, it was suggested by Khalil [11] that the contribution of plastic deformations to the pinning energy could lead to a logarithmic divergence of $U(J)$ (assuming that the interaction potential increases logarithmically), in good agreement with various relaxation data obtained in $\mathrm{YBaCuO}$ [7]. Alternatively, it has been suggested by Fisher et al. [12] that power divergent barriers (with low $\mu$ values) should also exist in the so-called vortex glass state and Blatter et al. [1] proposed that a renormalization of the shear modulus in the presence of dislocations could lead to a reduction of the $\mu$ exponent down to $\mu \sim 1 / 4$. Similarly Kierfeld et al. [13] recently extended the collective creep theory to topologically disordered vortex solids. They have shown that plastic vortex creep could still be described in terms of driven thermally activated dislocations motion associated with power law diverging barriers, $U_{p l}^{c}(j) \propto j^{-\mu}$, where $\mu$ here depends on the dislocation bundle size (being of the order of 1 for single dislocations and $0.4-0.5$ for dislocation bundles).

With the experimental point of view, the determination of the current dependence of the activation barrier is a complicated issue. The energy barrier is often deduced from magnetic relaxation data for which the time dependence of the current density can be related to $U$ through $U[J(t)]=k T \ln \left(t / t_{0}\right)$, where $t_{0}$ is a macroscopic time scale of the order of $10^{-6} \mathrm{~s}$ (for a review, see [7]). The collective creep theory thus corresponds to a nonlogarithmic decay of the current density $J(t) \propto\left[\ln \left(t / t_{0}\right)\right]^{-1 / \mu}$. However, clear deviations from a logarithmic decay are visible only after several time decades [4] and the measurements rapidly become prohibitively time consuming (the initial time for magnetic relaxation measurements is of the order of $1 \mathrm{~s}$, and a five decade measurement thus lasts for one day). An alternative - and completely equivalent - method to study the vortex dynamics is to measure the frequency dependence of the vortex response to an alternative field [14].

We will show here that, in $(\mathrm{K}, \mathrm{Ba}) \mathrm{BiO}_{3}$, the creep mechanism is collective on both sides of the orderdisorder transition line. At high temperature and/or high magnetic field, $\mu$ decreases, in good agreement with the plastic collective creep theory recently proposed by Kierfeld et al. [13]. We suggest that this decrease becomes visible only when the distance between dislocations in the disordered phase becomes smaller than the creeping 
vortex-bundle size, and large $\mu$ values can thus still be observed above the fishtail peak at low temperature. Most of the measurements were performed on high quality $(\mathrm{K}, \mathrm{Ba}) \mathrm{BiO}_{3}$ single crystals grown by electrocrystallization $\left(T_{c} \sim 31.2 \mathrm{~K}\right)$. Those particularly homogeneous crystals present very sharp superconducting transitions in both transport $\left(\Delta T_{c} \sim 0.15 \mathrm{~K}\right)$ and ac susceptibility $\left(\Delta T_{c} \sim 0.2 \mathrm{~K}\right.$ for $\left.h_{\mathrm{ac}}<0.01 \mathrm{G}\right)$ measurements. The high quality of the samples was futher confirmed by magneto-optical images as well as specific heat measurements. Its perfectly isotropic structure (i.e., cubic) then makes this system particularly well adapted to probe the various creeping models, avoiding any further complication related to the anisotropy.

In order to extend the frequency range, the ac response has been measured by means of two complementary techniques. At low frequency (i.e., for $0.2 \mathrm{~Hz}<f<$ $2 \mathrm{kHz}$ for $0.5 \mathrm{~T}<H_{\mathrm{dc}}<5 \mathrm{~T}$ and even $0.03 \mathrm{~Hz}<$ $f<20 \mathrm{kHz}$ for $H_{\mathrm{dc}}=0.1 \mathrm{~T}$ ), the broadband transmittivity $T_{H}\left(h_{\mathrm{ac}}, f, T\right)=\left[B\left(h_{\mathrm{ac}}, f, T\right)-B\left(h_{\mathrm{ac}}, f, T \gg\right.\right.$ $\left.\left.T_{c}\right)\right] /\left(B\left(h_{\mathrm{ac}}, f, T \gg T_{c}\right)-B\left(h_{\mathrm{ac}}, f, T \ll T_{c}\right)\right]$ was determined using a Hall probe. At higher frequency (i.e., $1<f<60 \mathrm{kHz}$ ), the ac susceptibility $\chi$ has been deduced from the change in the inductance of a miniature $\mathrm{Cu}$ secondary coil placed on the top of the sample. In the non-Ohmic regime, the ac response is related to the current density $j$ through $\chi\left(h_{\mathrm{ac}}, \omega, T\right)$ [or, equivalently, $\left.T_{H}\left(h_{\mathrm{ac}}, \omega, T\right)\right]=F\left[h_{\mathrm{ac}} / j(\omega, T)\right]$, where $F(x)$ depends on the pinning mechanism [14], the sample geometry and the flux creep exponent [15]. $F(x)$ was determined following the procedure proposed by Pasquini et al. [16] [note that $F(x)$ is frequency dependent and has thus been determined for every frequency] and $j(T, \omega)$ was then deduced by inverting $F(x)$ at fixed $h_{\mathrm{ac}}(\sim 5 \mathrm{G})$ : $j(T, \omega) \sim h_{\mathrm{ac}} / F^{-1}[\chi(T, \omega)]$. A characteristic curve obtained at $H_{\mathrm{dc}}=0.1 \mathrm{~T}$ and $T=28 \mathrm{~K}$ is shown in Fig. 1. As shown, the two sets of data nicely coincide on the common frequency range. $j(\omega)$ clearly deviates from a simple logarithmic dependence at high frequency and the solid line is a fit to the data using the collective creep formula,

$$
j(\omega, T)=\frac{j_{0}}{\left[\frac{k T}{U_{0}} \ln \left(\omega_{0} / \omega\right)\right]^{1 / \mu}}
$$

which yields to $\mu=5 / 2$ as expected in the small (vortex-)bundle regime [1] ( $j_{0}$ and $U_{0}$ are characteristic current and energy scales, respectively; see below). Note that the measurements were performed on a very large frequency range: $10^{-7}<\omega / \omega_{0}<1\left(\omega_{0} \sim 10^{6} \mathrm{rad} / \mathrm{s}\right.$; see below), whereas classical dc relaxation measurements would typically give access to a time window of the order of $10^{-9}<t_{0} / t<10^{-5}$ (schematically represented by the rectangle in Fig. 1). The ac measurements thus allow us to extend the experimental window towards large $\omega / \omega_{0}$

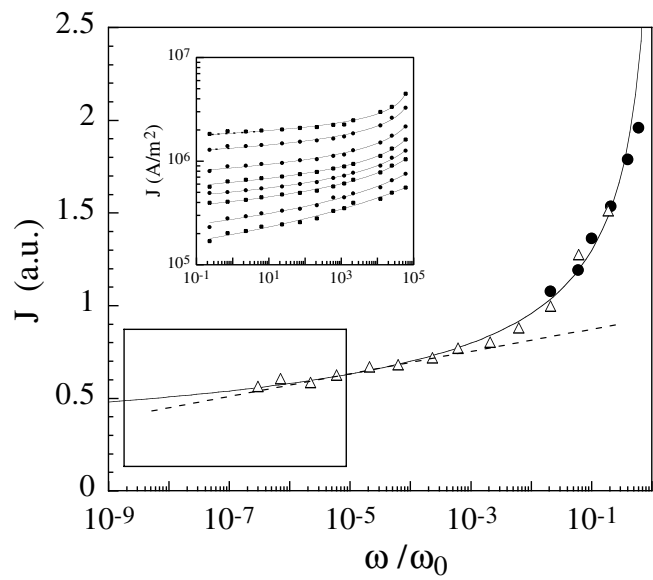

FIG. 1. Frequency dependence of the current density deduced from ac susceptibility measurements using a Hall probe (closed circles) and an induction technique (open triangles) at $T=$ $28 \mathrm{~K}$ and $H_{\mathrm{dc}}=0.1 \mathrm{~T}$. The rectangle schematically represents the time window usually accessible through dc magnetic relaxation measurements. The solid line is a fit to the data using Eq. (1) with $\mu=2.5$. Inset: frequency dependence of the current density deduced from ac susceptibility measurements at $H_{\mathrm{dc}}=2 \mathrm{~T}$ and $T(\mathrm{~K})=$ (from top to bottom) 24.5, 24.3, 24, $23.8,23.5,23,22$, and 21 . The solid lines are fits to the data using Eq. (1).

values for which the deviation from the logarithmic decay becomes clearly visible.

The value of the $\mu$ coefficient can also be directly obtained by calculating the inverse creep rate $S^{-1}\left(\omega_{n}\right)=$ $(d \ln j / d \ln \omega)^{-1}=\ln \left(\frac{\omega_{n+1}}{\omega_{n-1}}\right) / \ln \left(\frac{j_{n+1}}{j_{n-1}}\right)$. As shown in Fig. 2, at high temperature, $S^{-1}$ varies linearly with $\ln \left(\omega_{0} / \omega\right)$ with a negative slope $-\mu \sim-5 / 2$. At low temperature and low frequency we observed for all magnetic fields a small deviation from the collective creep behavior towards a frequency independent inverse creeping rate (see dashed line in Fig. 2) which would correspond to a logarithmic activation energy. For low dc fields (i.e., 0.1 and $0.5 \mathrm{~T}$ ) we did not observe any significant change in the $\mu$ coefficient with temperature [see Fig. 3(a)]. This observation is consistent with our small angle neutron scattering (SANS) experiments [17] which have shown that the vortex solid is well ordered at low temperature and low magnetic field. SANS experiments also confirmed that the ordered phase transforms into a disordered glass above some characteristic field $B^{*}(T)$ lying close to the fishtail peak. The transition line has then been estimated from the $J(H)$ curves, and the as-deduced $B^{*}(T)$ values have been reported in Fig. 3(b). Typical $j(\omega)$ curves obtained at $H_{\mathrm{dc}}=2 \mathrm{~T}$ for several temperatures in the vicinity of the transition line are shown in the inset of Fig. 1. The corresponding $\mu$ values (together with those obtained for $H_{\mathrm{dc}}=0.1,0.5$, and $5 \mathrm{~T}$ ) are reported in Fig. 3(a). As shown, at $2 \mathrm{~T}, \mu$ rapidly decreases at high temperature. Indeed, as $T$ becomes larger then the order-disorder transition temperature $T^{*}(H)$, dislocations 


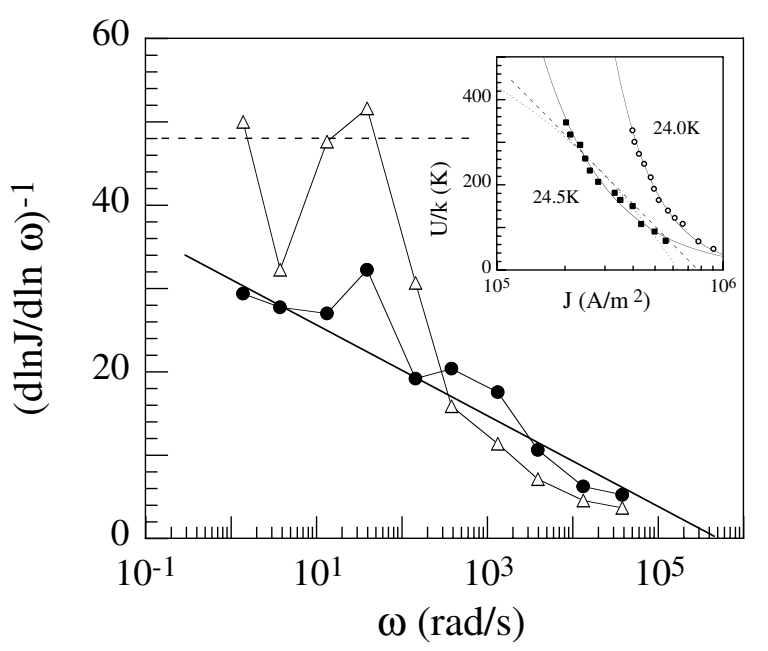

FIG. 2. Inverse creep rate $S^{-1}=(d \ln j / d \ln \omega)^{-1}$ versus $\ln (\omega)$ at $T=28 \mathrm{~K}$ (closed circles) and $T=26 \mathrm{~K}$ (open triangles) $\left(H_{\mathrm{dc}}=0.1 \mathrm{~T}\right)$. The solid line corresponds to the linear variation expected in the collective creep theory (with $\mu=5 / 2$ ). Some deviation from this theory towards a frequency independent creep rate is visible at low temperature and low frequency (dashed line). Inset: current dependence of the activation energy at $H_{\mathrm{dc}}=2 \mathrm{~T}$. The solid, dashed, and dotted lines are, respectively, $U \sim\left(J_{0} / J\right)^{\mu}, U \sim \ln \left(J / J_{0}\right)$, and $U \sim\left[1-\left(J / J_{0}\right)^{0.5}\right]$ fits to the data.

proliferate into the samples and this decrease is in good agreement with recent theoretical predictions [13] which suggested that $\mu=10 / 21<2.5$ for dislocation bundles in the so-called random manifold regime. This regime is equivalent to the vortex-bundle regimes in the ordered phase, and a very similar value $(\mu=2 / 5)$ has been predicted for very large dislocation bundle sizes (so-called Bragg glass regime).

Fits to the $j(\omega)$ data using Eq. (1) provide a very reasonable characteristic frequency $\omega_{0} \sim 10^{6}-10^{7} \mathrm{rad} / \mathrm{s}$ (see, for instance, Fig. 2 in which $\omega_{0}$ corresponds to $1 / S=0$ ). This value can then be used to calculate the current dependence of the creeping barrier $U(J)=k T \ln \left(\omega / \omega_{0}\right)$. Typical curves for $H_{\mathrm{dc}}=2 \mathrm{~T}$ are shown in the inset of Fig. 2. As shown, the logarithmic barrier model (dashed line) and the noncollective plastic barrier model (dotted line) yield very poor fits to the data which, in contrast, can be very well described by the collective creep theory (with $\mu \sim 1.3$ and 1.9 for $T=24.5$ and $24 \mathrm{~K}$, respectively). This unambiguously shows that the collective creep model still has to be used in the regime where $\mu$ decreases, as previously suggested by Klein et al. [18].

Surprisingly, for $H_{\mathrm{dc}}=5 \mathrm{~T}$, i.e., well above the orderdisorder transition field, $\mu$ still increases towards the $5 / 2$ value as the temperature decreases. It has been suggested by Kierfeld et al. [19] that the mean distance between two dislocations in the glassy state is $\sim R_{a}$, where $R_{a}$ is the length on which typical vortex displacements are of the order of the lattice spacing $a_{0}$. On the other hand, the dimension of the creeping vortex bundle is of the or-

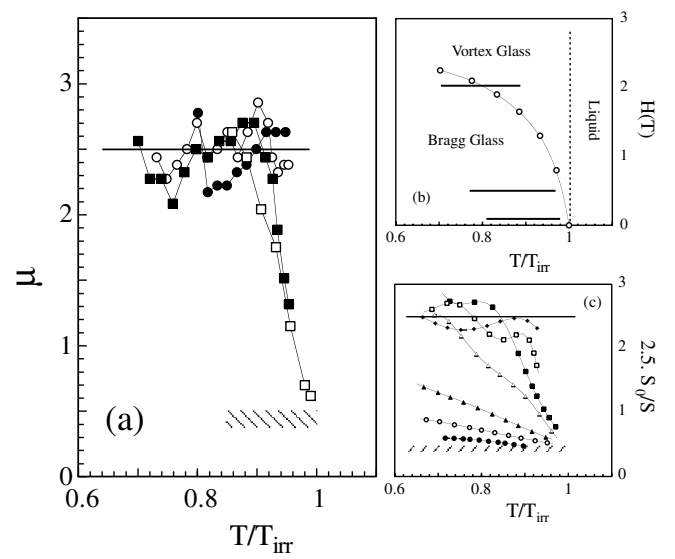

FIG. 3. (a) Creep exponent $\mu$ as a function of $T / T_{i r r}$ [where $T_{i r r}$ is the irreversibility temperature $=T_{c}\left(1-\left[H / H_{0}\right]^{0.7}\right)$ with $H_{0} \sim 24 \mathrm{~T}$ and $\left.T_{c}=31.2 \mathrm{~K}\right]$ at $H=5 \mathrm{~T}$ (open squares), $2 \mathrm{~T}$ (closed squares), $0.5 \mathrm{~T}$ (open circles), and $0.1 \mathrm{~T}$ (closed circles) in an optimally doped $(\mathrm{K}, \mathrm{Ba}) \mathrm{BiO}_{3}$ sample $\left(T_{c} \sim 31.2 \mathrm{~K}\right)$. The hatched area corresponds to the dislocation creep limit and the horizontal line corresponds to the elastic collective creep limit in the small (vortex-)bundle regime. (b) Temperature dependence of the fishtail position in the same sample. The horizontal lines indicate the temperature range for which a constant $\mu$ value $\sim 5 / 2$ has been measured. (c) Temperature dependence of the renormalized inverse creeping rate $\left(S_{0} \sim 5 \%\right)$ in a nonoptimally doped sample $\left(T_{c}=23 \mathrm{~K}\right)$ at $H(\mathrm{~T})=8,7,6,4,2,0.5$, and 0.2 (from bottom to top).

der of $R_{\perp} \sim \xi\left(j_{0} / j\right)^{1 / 2}$ [ $j_{0}$ being the depairing current and $\xi$ the coherence length $\sim 30 \AA$ at low temperature in $\left.(\mathrm{K}, \mathrm{Ba}) \mathrm{BiO}_{3}\right]$. At low temperature (and not too high magnetic field) $R_{\perp}$ is of the order of a few times $a_{0}$ for realistic $j_{0} / j$ values $\sim 100-1000$, whereas $R_{a} \sim 10-100 a_{0}$ [3]. The vortex-bundle size could hence be much smaller than the distance between dislocations in some part of the disordered phase, thus explaining why the creep can still be described by the elastic model above the transition. As $T$ increases, $R_{a}$ tends towards $R_{\perp}$, and $\mu$ finally decreases at high temperature (as the creep becomes plastic).

As $R_{\perp}$ increases with field, whereas $R_{a}$ decreases [3], plastic collective creep is finally expected to be observed at all temperatures for large magnetic fields. As pointed out above, in the collective creep model, the inverse creeping rate $S^{-1}$ is directly proportional to $\mu$. Figure 3(c) shows the temperature dependence of $S^{-1}$ assuming that $\mu=2.5$ at low temperature and low field [i.e., taking a very reasonable $\ln \left(\omega / \omega_{0}\right)=6.4$ value for $\left.\omega=213 \mathrm{~Hz}\right]$ in a nonoptimally doped sample $\left(T_{c} \sim 23 \mathrm{~K}\right)$. As expected, at high field the creep is plasticlike on the entire temperature range with $\mu \sim 0.5$ in excellent agreement with the predictions of Kierfeld et al. [13]. Similar behavior will probably show up in the optimally doped sample but apparently for higher magnetic fields.

The last parameter in Eq. (1) is $j_{0}\left(\frac{U_{0}}{k T}\right)^{1 / \mu} \cdot j_{0} U_{0}^{1 / \mu}$ can then be deduced from the temperature dependence of the current density at fixed frequency $j_{0} U_{0}^{1 / \mu} \propto j T^{1 / \mu}$. The 


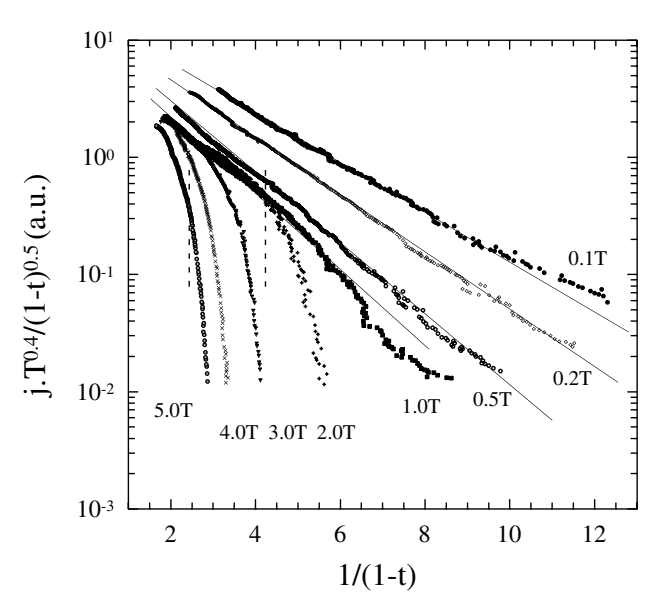

FIG. 4. $j T^{0.4} /(1-t)^{0.5}$ as a function of $1 /(1-t)$ (with $t=$ $\left.T / T_{c}\right)$. The solid lines are guides to the eye corresponding to the expected variation for $B>B_{s b}$ [Eq. (2)]. The dashed vertical lines indicate the temperatures above which $\mu$ starts to decrease at 5 and $2 \mathrm{~T}$ (see Fig. 3).

small (vortex-)bundle creep regime can be reached by two different ways, depending on the strength of the magnetic field: for $B<B_{s b}$ this regime is observable only for current densities smaller than some characteristic crossover value $j_{s b}\left(j_{0}=j_{s b}\right.$ and $\left.U_{0}=U_{s b}\right)$, whereas for $B_{s b}<$ $B<B_{l b}$ [where $B_{l b}$ is the crossover field towards the large (vortex-)bundle regime] this regime is expected to be visible right below the critical current $j_{c}: j_{0}=j_{c}$ and $U_{0}=U_{c}$ [1]. The temperature (and magnetic field) dependence of $j_{0} U_{0}^{1 / \mu}$ is indeed very different in both cases. For $\delta T_{c}$ pinning (i.e., pinning induced by fluctuations in the critical temperature), one obtains $j_{s b} U_{s b}^{1 / \mu} \sim(1-$ $t)^{43 / 50}$ and

$$
j_{c} U_{c}^{1 / \mu} \sim(1-t)^{1 / 2} \exp \left(\frac{-\alpha(B)}{1-t}\right)
$$

for $B<B_{s b}$ and $B>B_{s b}$, respectively $\left(t=T / T_{c}\right.$ and $\alpha(B)$ is related to the magnetic field dependence of the elastic moduli). As shown in Fig. 4, the experimental data are in excellent agreement with Eq. (2) for $0.1<H_{\mathrm{dc}}<$ $1 \mathrm{~T}$. Note that, in contrast with the classical elastic theory, $\alpha$ is only weakly magnetic field dependent in the vicinity of the peak effect, probably reflecting some renormalization of the elastic constants. As expected, for higher magnetic field, clear deviations from Eq. (2) can be observed as soon as $\mu$ starts to decrease (see dashed lines at 2 and $5 \mathrm{~T}$ ). As shown in [18], the temperature (and magnetic field) dependence of the current density is then mainly governed by $\mu(T, H)$.

In conclusion, we have shown that vortex dynamics in $(\mathrm{K}, \mathrm{Ba}) \mathrm{BiO}_{3}$ is well described by the collective creep theory in the ordered and disordered states. We suggest that the creep mechanism remains elastic $(\mu \sim 5 / 2)$ in the disordered phase as long as the size of the creeping vortex bundle is smaller than the mean distance between dislocations. At higher magnetic field and/or temperature, $\mu$ decreases, as predicted recently by Kierfeld et al. for plastic collective creep [13].

We thank Dr. T. Giamarchi for very interesting discussions.

[1] G. Blatter, M. V. Feigel'man, V. B. Geshkenbein, A. I. Larkin, and V.M. Vinokur, Rev. Mod. Phys. 66, 1125 (1994).

[2] M. V. Feigel'man, V.B. Geshkenbein, A. I. Larkin, and V. M. Vinokur, Phys. Rev. Lett. 63, 2303 (1989).

[3] T. Giamarchi and P. Le Doussal, Phys. Rev. B 52, 1530 (1994); T. Giamarchi and P. Le Doussal, Phys. Rev. B 55, 6577 (1997).

[4] J. R. Thompson, Yan Ren Sun, and F. Holtzberg, Phys. Rev. B 44, 458 (1991).

[5] C. J. van der Beek, S. Colson, M. Konczykowski, M. V. Indenbom, R. J. Drost, and P. H. Kes, Physica (Amsterdam) 341C-348C, 1279 (2000).

[6] D. Fuchs, R. A. Doyle, E. Zeldov, S. F. W. Rycroft, T. Tamegai, S. Ooi, M. L. Rappaport, and Y. Myasoedov, Phys. Rev. Lett. 81, 3944 (1998).

[7] Y. Yeshurun, A. P. Malozemoff, and A. Shaulov, Rev. Mod. Phys. 68, 911 (1996).

[8] D. Ertas and D. Nelson, Physica (Amsterdam) 272C, 79 (1997); V. M. Vinokur et al., Physica (Amsterdam) 295C, 209 (1998).

[9] Y. Abulafia, A. Shaulov, Y. Wolfus, R. Prozorov, L. Burlachkov, Y. Yeshurun, D. Majer, E. Zeldov, H. Wuhl, V. B. Geshkenbein, and V. M. Vinokur, Phys. Rev. Lett. 77, 1596 (1996).

[10] T. Aouaroum et al., Physica (Amsterdam) 294C, 42 (1998); T. Aouaroum et al., Physica (Amsterdam) 306C, 238 (1998); M. Pissas et al., Phys. Rev. B 59, 12121 (1999); D. Stamopoulos et al., Physica (Amsterdam) 317C, 658 (1999).

[11] A. E. Khalil, Phys. Lett. A 246, 353 (1998).

[12] D. Fisher et al., Phys. Rev. B 43, 130 (1991); D. Fisher and T. Natterman, Phys. Rev. B 43, 10372 , (1991).

[13] J. Kierfeld, H. Nordborg, and V. M. Vinokur, Phys. Rev. Lett. 85, 4948 (2000).

[14] C. J. van der Beek et al., Phys. Rev. B 48, 3393 (1993); C. J. van der Beek et al., Physica (Amsterdam) 258C, 105 (1996).

[15] E. H. Brandt, Phys. Rev. B 55, 14513 (1997), and references therein.

[16] G. Pasquini, L. Civale, H. Lanza, and G. Nieva, Phys. Rev. B 59, 9627 (1999).

[17] I. Joumard, J. Marcus, T. Klein, and R. Cubitt, Phys. Rev. Lett. 82, 4930 (1999).

[18] T. Klein et al., Phys. Rev. Lett. 79, 3795 (1997); T. Klein et al., Europhys. Lett. 42, 79 (1998).

[19] J. Kierfeld, Physica (Amsterdam) 300C, 171 (1998); J. Kierfeld and V. M. Vinokur, Phys. Rev. B 61, R14928 (2000). 